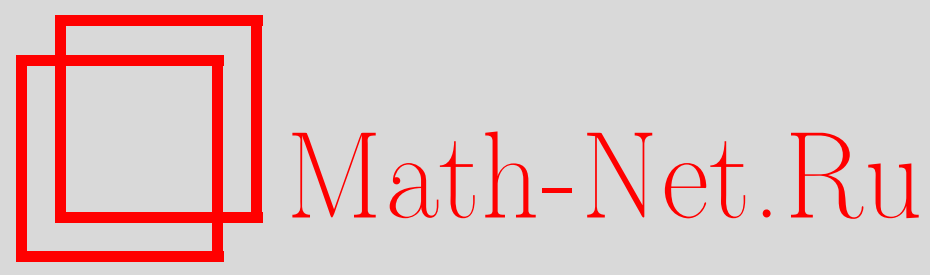

В. Н. Старовойтов, О движении двухкомпонентной жидкости при наличии капиллярных сил, Матем. заметки, 1997, том 62, выпуск 2, 293-305

DOI: https://doi.org/10.4213/mzm1611

Использование Общероссийского математического портала Math-Net.Ru подразумевает, что вы прочитали и согласны с пользовательским соглашением http://www . mathnet.ru/rus/agreement

Параметры загрузки:

IP : 3.80 .181 .102

26 апреля 2023 г., 11:59:10

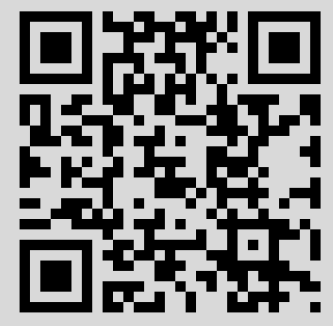




\title{
О ДВИЖЕНИИ ДВУХКОМПОНЕНТНОЙ ЖИДКОСТИ ПРИ НАЛИЧИИ КАПИЛЛЯРНЫХ СИЛ
}

\author{
В.Н. Старовойтов
}

В работе исследуется качественное поведение при $t \rightarrow \infty$ решения задачи Коши для системы уравнений, описьвающей движение вязкой двухкомпонентной жидкости. При этом в используемой модели учитывается как взаимная дифффузия компонент жидкости, так и их капиллярное взаимодействие. Описано $\omega$-предельное множество траекторий динамической системы, порожденной задачей. Показано, что стационарное решение задачи, представляющее собой однородное неподвижное распределение одной из компонент, является асимптотически устойчивып. Любое другое стационарное решение не является асимптотически устойчивым и неустойчиво, если вблизи него нет стационарных решений с меньшим уровнем энергии.

Библиография: 15 названий.

Постановка задачи. Как известно, разрешимость задачи о движении двух несмешивающихся жидкостей, разделенных границей, обладающей поверхностным натяжением, в классической постановке доказана лишш в “малом". В связи с этим в работе [1] для описания этого явления была предложена следующая модель, построенная на основе идей Ван дер Ваальса [2], Кортевега [3], Кана и Хилларда [4]:

$$
\begin{gathered}
\bar{v}_{t}+(\bar{v} \cdot \nabla) \bar{v}=-\nabla p+\Delta \bar{v}-\operatorname{div}(\nabla \varphi \otimes \nabla \varphi), \\
\operatorname{div} \bar{v}=0, \\
\varphi_{t}+\bar{v} \cdot \nabla \varphi=\Delta\left(-\Delta \varphi+W^{\prime}(\varphi)\right) .
\end{gathered}
$$

В данной модели учитьваются как капиллярное взаимодействие жидкостей, так и их взаимная диффузия. Функции, входящие в уравнения, имеют такой физический смысл: $\bar{v}$ - вектор скорости, $p$ - давление, $\varphi$ - концентрация одной из компонент жидкости, $W^{\prime}(\varphi)=d W / d \varphi$, где функция $W(\varphi)$ - так назьваемьй "double-well”-потенциал. Вообще говоря, по своему физическому смыслу концентрация $\varphi$ неможет принимать значения вне отрезка $[0,1]$, чего можно добиться подходящим выбором функции $W$. В данной статье мы будем пренебрегать этим условием и считать, что $\varphi$ может принимать любые значения на числовой оси. Относительно функции $W$ будем предполагать вьполненными следующие условия:

1) $W \in C^{3}(\mathbb{R})$;

Работа выполнена при поддержке Международного научного фонда, грант RCK300. 
2) $W^{\prime}(\xi)<0$ при $\xi \in(-\infty, 0) \cup(1 / 2,1), W^{\prime}>0$ при $\xi \in(0,1 / 2) \cup(1, \infty), W(0)=$ $W(1)=0, W(\xi) \geqslant 0, \xi \in \mathbb{R}$

3) $\lim _{|\xi| \rightarrow \infty} \frac{W(\xi)}{|\xi|^{2}}=\infty$.

Всем этим условиям удовлетворяет функция $W(\varphi)=\varphi^{2}(\varphi-1)^{2}$. Для определенности будем считать, что в уравнении (3) фигурирует именно эта функция.

В данной работе исследуется задача Коши для системы (1)-(3) в случае двух пространственных переменных: $\bar{x}=\left(x_{1}, x_{2}\right) \in \mathbb{R}^{2}, \bar{v}=\left(v_{1}, v_{2}\right) \in \mathbb{R}^{2}$. Зададим начальные условия:

$$
\bar{v}(\bar{x}, 0)=\bar{v}_{0}(\bar{x}), \quad \varphi(\bar{x}, 0)=\varphi_{0}(\bar{x}) .
$$

В статье будем использовать следующие обозначения:

$\boldsymbol{L}_{p}(\Omega)=\left\{\bar{u}=\left(u_{1}, u_{2}\right) \mid u_{i} \in L_{p}(\Omega), i=1,2\right\}$

$J(\Omega)=\left\{\bar{u} \in C_{0}^{\infty}(\Omega) \mid \operatorname{div} \bar{u}=0\right\}$

$J X(\Omega)$ - замькание $J(\Omega)$ по норме пространства $X(\Omega)$;

$L_{p}=L_{p}\left(\mathbb{R}^{2}\right), \boldsymbol{L}_{p}=\boldsymbol{L}_{p}\left(\mathbb{R}^{2}\right) ;$

$H_{p}^{s}$-пространство бесселевых потенщиалов [5], $H_{p}^{0}=L_{p}, H^{s}=H_{2}^{s}$;

$\|\cdot\|_{p}-$ норма в $\boldsymbol{L}_{p}$ и $L_{p} ;$

$\|\cdot\|_{s, p}-$ норма в $H_{p}^{s}$;

$\|\cdot\|,(\cdot, \cdot)$ - норма и скалярное произведение в $\boldsymbol{L}_{2}$ и $L_{2}$;

$P$ - оператор ортогонального проектирования в $\boldsymbol{L}_{2}$ на $J \boldsymbol{L}_{2}$;

$A$ - самосопряженное расширение по $Ф$ ридрихсу оператора $P(-\Delta+I)$ в $J \boldsymbol{L}_{2} \mathrm{c}$ областью определения $D(A)=H^{2}$;

$B$ - самосопряженное расширение по $\Phi$ ридрихсу оператора $\left(\Delta^{2}+I\right)$ в $L_{2}$ с областью определения $D(B)=H^{4}$;

$I$ - тождественньй оператор.

Запишем задачу (1)-(4) в виде задачи Коши для системы уравнений в пространстве $J \boldsymbol{L}_{2} \times L_{2}$

$$
\begin{gathered}
\bar{v}_{t}+A \bar{v}=\bar{f} \equiv \bar{v}-P(\bar{v} \cdot \nabla) \bar{v}-P \operatorname{div}(\nabla \varphi \otimes \nabla \varphi) \\
\varphi_{t}+B \varphi=g \equiv \varphi-\bar{v} \cdot \nabla \varphi+\Delta W^{\prime}(\varphi) \\
\bar{v}(0)=\bar{v}_{0}, \quad \varphi(0)=\varphi_{0}
\end{gathered}
$$

Здесь $\bar{v}:(0, \infty) \rightarrow J \boldsymbol{L}_{2}, \varphi:(0, \infty) \rightarrow L_{2}$.

ОПРеДЕЛЕниЕ. Решением задачи (5)-(7) назовем пару функций $\bar{v}:[0, \infty) \rightarrow J \boldsymbol{L}_{2}$, $\varphi:[0, \infty) \rightarrow L_{2}$ таких, что $\bar{v}(t) \in D(A), \varphi(t) \in D(B), \bar{v}_{t}(t) \in \boldsymbol{L}_{2}, \varphi_{t}(t) \in L_{2}$ для почти всех $t \in(0, \infty)$, непрерывных по $t$ в $J \boldsymbol{L}_{2}$ и $L_{2}$ соответственно и удовлетворяющих уравнениям (5), (6) и начальным условиям (7).

В п. 1 данной статьи доказывается однозначная разрешимость задачи (5)-(7). Далее, в п. 2 изучается поведение решения при $t \rightarrow \infty$. Пункт 3 посвящен исследованию устойчивости стационарных решений задачи. 
1. Корректность задачи. Нам понадобятся некоторые свойства пространств бесселевых потенциалов $\left(\right.$ в $\left.\mathbb{R}^{2}\right)[5]$ :

$$
\begin{gathered}
H_{p}^{s} \subset H_{q}^{r} \quad \text { при } 1<p \leqslant q<\infty, \quad s-\frac{2}{p} \geqslant r-\frac{2}{q} ; \\
H_{p}^{2 / p+r} \subset C^{r}, \quad 1<p<\infty, \quad r>0, \quad r \text { нецелое; } \\
\|u\|_{s, p} \leqslant c_{\theta}\|u\|_{s_{0}, p_{0}}^{1-\theta} \cdot\|u\|_{s_{1}, p_{1}}^{\theta},
\end{gathered}
$$

где $-\infty<s_{0}, s_{1}<\infty, 1<p_{0}, p_{1}<\infty, 0<\theta<1, s=(1-\theta) s_{0}+\theta s_{1}, 1 / p=(1-\theta) / p_{0}+\theta / p_{1}$, $c_{\theta}$ - константа, зависяшая от $\theta$.

Докажем сначала одну оценку, которая является обобщением оценки П. Е. Соболевского [6].

ПРЕДЛОЖЕНИЕ 1. Справедливо неравенство

$$
\left\|A^{-\alpha}(u v)\right\| \leqslant C\|u\|_{1 / \alpha} \cdot\|v\|,
$$

где $0<\alpha<1 / 2$, константа $C$ не зависит от $u$ u

ДОКАЗАТЕЛЬСТво. В силу (8)

$$
\|\psi\| \leqslant C\left\|A^{\alpha} \psi\right\|_{p}, \quad p=\frac{2}{2 \alpha+1},
$$

для любой функции $\psi \in H_{p}^{2 \alpha}$.

Поэтому $\left\|A^{-\alpha}(u v)\right\| \leqslant C\|u v\|_{p}$. Отсюда, используя неравенство Гёльдера, получим (10).

TEорема 1. Пусть $\bar{v}_{0} \in H^{\alpha}, \varphi_{0} \in H^{\beta}, 1<\alpha<2,2<\beta<4$. Тогда существует единственное решение задачи (5)-(7) такое, что $\bar{v} \in C\left(0, \infty ; J H^{\alpha}\right) \cap L_{2}\left(0, \infty ; H^{2}\right)$, $\varphi \in C\left(0, \infty ; H^{\beta}\right)$.

ДокАЗАтЕльСтво. Докажем сначала локальную разрешимость задачи. Согласно теореме 3.3.3 [7] достаточно проверить локальную липшицевость функции

$$
F=\left(\begin{array}{l}
\bar{f} \\
g
\end{array}\right): H^{\alpha} \times H^{\beta} \rightarrow \boldsymbol{L}_{2} \times L_{2}
$$

Легко проверить, что

$$
\|F\|=\sqrt{\|\bar{f}\|^{2}+\|g\|^{2}} \leqslant\|f\|+\|g\| \leqslant C\left(\|\bar{\varphi}\|_{\beta, 2}^{2}+\|\varphi\|_{2,2}^{3}+\|\bar{v}\|_{\alpha, 2}^{2}\right) .
$$

Так как $F$ - полиномиальное отображение, то в силу полученной оценки оно локально лишшицево. Таким образом, доказано, что если $\bar{v}_{0} \in J H^{\alpha}, \varphi_{0} \in H^{\beta}$, то задача (5)-(7) локально однозначно разрешима. Причем на всем промежутке времени сушествования решения $[0, T] \bar{v}(t) \in D(A), \varphi(t) \in D(B), \bar{v} \in C\left(0, T ; J H^{\alpha}\right), \varphi \in C\left(0, T ; H^{\beta}\right)$.

Для доказательства глобальной однозначной разрешимости при $t \in(0, \infty)$ согласно следствию 3.3 .5 [7] достаточно показать, что, пока решение существует, имеет место оценка

$$
\|F\| \leqslant C\left(1+\|\bar{v}\|_{\alpha, 2}+\|\varphi\|_{\beta, 2}\right)
$$


где постоянная $C$ не зависит от $t$. Далее все константы, не зависящие от $t$, мы будем обозначать буквой $C$.

Введем обозначение $\theta=-\Delta \varphi+W^{\prime}(\varphi)$. Для решения задачи (5)-(7) имеет место энергетическое тождество

$$
\frac{d}{d t}\left(\frac{1}{2}\|\bar{v}(t)\|^{2}+\frac{1}{2}\|\nabla \varphi(t)\|^{2}+\int_{\mathbb{R}^{2}} W(\varphi(t)) d \bar{x}\right)+\|\nabla \bar{v}(t)\|^{2}+\|\nabla \theta(t)\|^{2}=0 .
$$

Отсюда следует, что существует константа $C$ такая, что

$$
\|\bar{v}(t)\| \leqslant C, \quad t \geqslant 0 .
$$

Кроме того, так как для любой функции $u \in D\left(A^{1 / 2}\right)$ справедливо неравенство [8]

Tо

$$
\|u\|_{4}^{2} \leqslant \frac{1}{\sqrt{2}}\|u\| \cdot\|\nabla u\|,
$$

$$
\int_{0}^{t}\|v(s)\|_{4}^{4} d s \leqslant \frac{1}{2} \max _{t \geqslant 0}\|v(t)\|^{2} \int_{0}^{t}\|\nabla v(s)\|^{2} d s \leqslant C .
$$

Приступим к получению равномерных по $t$ оценок функции $\varphi$. Некоторые сложности возникают из-за того, что у нас пока нет оценки функции $\varphi(t)$ в $L_{2}$.

ЛЕмма 1.1. Пусть $\varphi(t)$ - решение задачи (5)-(7). Тогда

$$
\left\|B^{-1 / 8} \Delta W^{\prime}(\varphi)\right\| \leqslant C\|\varphi\|_{2,2} \text {. }
$$

ДОКАЗАТЕЛЬСТВО. Введем две функции:

$$
\varphi_{1}(\bar{x}, t)=\left\{\begin{array}{rr}
\varphi(\bar{x}, t), & 0 \leqslant \varphi(\bar{x}, t) \leqslant 2, \\
0, & \varphi(\bar{x}, t) \leqslant 0, \\
2, & \varphi(\bar{x}, t) \geqslant 2,
\end{array} \quad \varphi_{2}(\bar{x}, t)=\left\{\begin{array}{lr}
\varphi(\bar{x}, t)-2, & \varphi(\bar{x}, t) \geqslant 2, \\
\varphi(\bar{x}, t), & \varphi(\bar{x}, t) \leqslant 0, \\
0, & 0 \leqslant \varphi(\bar{x}, t) \leqslant 2 .
\end{array}\right.\right.
$$

Очевидно, что $\varphi=\varphi_{1}+\varphi_{2}$. Кроме того, $\varphi_{1} \in L_{\infty}$, а так как $W(\xi) \geqslant \xi^{2}$, если $\xi \notin(0,2)$, то в силу (12) $\varphi_{2} \in L_{2}$. Заметим также, что $\nabla \varphi_{i} \in L_{2}, i=1,2$. Отсюда и из (8) следует, что $\varphi_{2} \in L_{p}, 2 \leqslant p<\infty$.

Таким образом,

$$
\left\|\varphi_{1}\right\|_{\infty}+\left\|\nabla \varphi_{1}\right\|+\left\|\varphi_{2}\right\|_{p}+\left\|\nabla \varphi_{2}\right\| \leqslant C, \quad 2 \leqslant p<\infty .
$$

В силу выбора функции $W$

$$
\Delta W^{\prime}(\varphi)=12|\nabla \varphi|^{2}(2 \varphi-1)+2 \Delta \varphi\left(6 \varphi^{2}-6 \varphi+1\right) .
$$

Будем вьводить оценку (16) для каждого слагаемого по отдельности. Ниже будут постоянно использоваться оценки (8)-(10), (12) без ссылок на них:

$$
\begin{aligned}
\left\|B^{-1 / 8}|\nabla \varphi|^{2}\right\| & \leqslant C\|\nabla \varphi\|_{4} \cdot\|\nabla \varphi\| \leqslant C\|\nabla \varphi\|_{4} \leqslant C \|_{\varphi / 2,2}, \\
\left\|B^{-1 / 8}|\nabla \varphi|^{2} \varphi\right\| & \leqslant \sum_{i=1}^{2}\left\|B^{-1 / 8}|\nabla \varphi|^{2} \varphi_{i}\right\| \leqslant C\left\|\varphi_{1} \nabla \varphi\right\|_{4}+C\left\|\varphi_{2} \nabla \varphi\right\|_{4} \\
& \leqslant C\|\nabla \varphi\|_{4}+C\left\|\varphi_{2}\right\|_{8} \cdot\|\nabla \varphi\|_{8} \leqslant C\|\varphi\|_{7 / 4,2}, \\
\left\|B^{-1 / 8} \varphi^{2} \Delta \varphi\right\| & \leqslant\left\|B^{-1 / 8} \varphi_{1}^{2} \Delta \varphi\right\|+\left\|B^{-1 / 8} \varphi_{2}^{2} \Delta \varphi\right\|+2\left\|B^{-1 / 8} \varphi_{1} \varphi_{2} \Delta \varphi\right\| \\
& \leqslant C\left\|\varphi_{1}^{2} \Delta \varphi\right\|+C\left\|\varphi_{2}^{2}\right\|_{4} \cdot\|\Delta \varphi\|+C\left\|\varphi_{1} \varphi_{2}\right\|_{4} \cdot\|\Delta \varphi\| \leqslant C\|\Delta \varphi\| .
\end{aligned}
$$

Здесь был использован тот факт, что $B^{-1 / 8}$ - ограниченньй оператор в $L_{2}$.

Остальные слагаемые оцениваются аналогично. Из оценок (17) следует утверждение леммы. 
Лемма 1.2. Пусть $\varphi_{0} \in H^{\beta}, 2 \leqslant \beta<5 / 2$. Тогда

$$
\|\varphi(t)\|_{\beta, 2} \leqslant C
$$

әде постоянная $C$ зависит только от $\varphi_{0}, \bar{v}_{0}$.

ДоКАЗАТЕЛЬСТВО. В силу (6) для функции $\varphi(t)$ имеет место представление

$$
\varphi(t)=e^{-B t} \varphi_{0}+\int_{0}^{t} e^{-B(t-s)}\left(\varphi(s)+\Delta W^{\prime}(\varphi(s))-\bar{v}(s) \cdot \nabla \varphi(s)\right) d s .
$$

Отсюда

$$
\begin{aligned}
& \|\varphi(t)\|_{\beta, 2} \leqslant\left\|e^{-B t} \varphi_{0}\right\|_{\beta, 2}+\int_{0}^{t}\left\|e^{-B(t-s)}\left(\varphi+\Delta W^{\prime}(\varphi)-\bar{v} \cdot \nabla \varphi\right)\right\|_{\beta, 2} d s \\
& \leqslant\left\|e^{-B t} \varphi_{0}\right\|_{\beta, 2}+C \int_{0}^{t}\left\|B^{\beta / 4+1 / 8} e^{-B(t-s)} B^{-1 / 8}\left(\varphi+\Delta W^{\prime}(\varphi)-\bar{v} \cdot \nabla \varphi\right)\right\| d s \\
& \leqslant\left\|e^{-B t} \varphi_{0}\right\|_{\beta, 2}+C \int_{0}^{t}\left\|B^{\beta / 4+1 / 8} e^{-B(t-s)}\right\| \cdot\left\|B^{-1 / 8}\left(\varphi+\Delta W^{\prime}(\varphi)-\bar{v} \cdot \nabla \varphi\right)\right\| d s \\
& \leqslant\left\|e^{-B t} \varphi_{0}\right\|_{\beta, 2}+C \int_{0}^{t}(t-s)^{-\beta / 4-1 / 8} e^{-(t-s)}\left\|B^{-1 / 8}\left(\varphi+\Delta W^{\prime}(\varphi)-\bar{v} \cdot \nabla \varphi\right)\right\| d s .
\end{aligned}
$$

Используя (10), (12) и (15), оценим интеграл

$$
\begin{aligned}
& \int_{0}^{t}(t-s)^{-\beta / 4-1 / 8} e^{-(t-s)}\left\|B^{-1 / 8} \bar{v} \cdot \nabla \varphi\right\| d s \\
& \quad \leqslant C \int_{0}^{t}(t-s)^{-\beta / 4-1 / 8} e^{-(t-s)}\|\bar{v}\|_{4} \cdot\|\nabla \varphi\| d s \\
& \quad \leqslant C\left(\int_{0}^{t}(t-s)^{-4(\beta / 4+1 / 8) / 3} e^{-4(t-s) / 3} d s\right)^{3 / 4}\left(\int_{0}^{t}\|\bar{v}(s)\|_{4}^{4} d s\right)^{1 / 4}<C,
\end{aligned}
$$

если $\beta<5 / 2$.

Из (16), (19) и (20) следует

$$
\|\varphi(t)\|_{\beta, 2} \leqslant\left\|e^{-B t} \varphi_{0}\right\|_{\beta, 2}+C+C \int_{0}^{t}(t-s)^{-\beta / 4-1 / 8} e^{-(t-s)}\|\varphi(s)\|_{\beta, 2} d s
$$

при $2 \leqslant \beta<5 / 2$. Из этого неравенства и леммы Гронуолла получаем утверждение леммы.

Из леммы 1.2, в частности, следует, что

$$
\|\varphi(t)\|_{\infty} \leqslant C
$$

Теперь приступим к получению оценок для функции $\bar{v}$. Домножив (1) на $\Delta \bar{v},(3)-$ на $\Delta \bar{v} \cdot \nabla \varphi$, получим

$$
\frac{1}{2} \frac{d}{d t}\left\|\bar{v}_{x}\right\|^{2}+\|\Delta \bar{v}\|^{2}+((\bar{v} \cdot \nabla) \bar{v}, \Delta \bar{v})=(\nabla \theta, \varphi \Delta \bar{v}) .
$$


Здесь $\bar{v}_{x}-$ матрица первых производных функции $\bar{v}$. В силу (21)

$$
(\nabla \theta, \varphi \Delta \bar{v}) \leqslant C\|\nabla \theta\| \cdot\|\Delta \bar{v}\| \leqslant C^{2}\|\nabla \theta\|^{2}+\frac{1}{4}\|\Delta \bar{v}\|^{2}
$$

Для любой функции $u \in H^{2}$ имеет место оценка [8]

$$
\|u\|_{2,2}^{2} \leqslant \frac{3}{2}\left(\|u\|^{2}+\|\Delta u\|^{2}\right)
$$

Используя оценку (24), а также (14), получим

$$
\begin{aligned}
((\bar{v} \cdot \nabla) \bar{v}, \Delta \bar{v}) & \leqslant\|\Delta \bar{v}\| \cdot\|\bar{v}\|_{4} \cdot\left\|\bar{v}_{x}\right\|_{4} \leqslant C\|\Delta \bar{v}\| \cdot\|\bar{v}\|^{1 / 2} \cdot\left\|\bar{v}_{x}\right\| \cdot\left\|\bar{v}_{x x}\right\|^{1 / 2} \\
& \leqslant C\left(\|\Delta \bar{v}\| \cdot\|\bar{v}\| \cdot\left\|\bar{v}_{x}\right\|+\|\Delta \bar{v}\|^{3 / 2} \cdot\|\bar{v}\|^{1 / 2} \cdot\left\|\bar{v}_{x}\right\|\right) \\
& \leqslant \frac{1}{4}\|\Delta \bar{v}\|^{2}+C\left\|\bar{v}_{x}\right\|^{4}+C\left\|\bar{v}_{x}\right\|^{2} .
\end{aligned}
$$

Подстановка неравенств (23), (25) в (22) дает

$$
\frac{d}{d t}\left\|\bar{v}_{x}\right\|^{2}+\|\Delta \bar{v}\|^{2} \leqslant C\left\|\bar{v}_{x}\right\|^{4}+C\left\|\bar{v}_{x}\right\|^{2}+C\|\nabla \theta\|^{2} .
$$

Из этого неравенства с помощью леммы Гронуолла получим

$$
\begin{aligned}
\left\|\bar{v}_{x}(t)\right\|^{2} \leqslant & \left\|\bar{v}_{x}(0)\right\|^{2} \exp \left(C \int_{0}^{t}\left\|\bar{v}_{x}(s)\right\|^{2} d s\right) \\
& +C \exp \left(C \int_{0}^{t}\left\|\bar{v}_{x}(s)\right\|^{2} d s\right) \int_{0}^{t}\left(\left\|\bar{v}_{x}(s)\right\|^{2}+\|\nabla \theta(s)\|^{2}\right) d s .
\end{aligned}
$$

Отсюда в силу (12) следует, что

$$
\left\|\bar{v}_{x}(t)\right\| \leqslant C, \quad t \geqslant 0
$$

если $\bar{v}_{0} \in H^{1}$. Кроме того, из $(12),(26)$ и $(27)$ имеем

$$
\int_{0}^{t}\|\Delta \bar{v}(s)\|^{2} d s \leqslant C, \quad t \geqslant 0
$$

Теперь у нас есть все необходимое для получения оценки (11). Нетрудно видеть, что

$$
\begin{gathered}
\|F\| \leqslant\|\bar{v}\|+\|\varphi\|+\|(\bar{v} \cdot \nabla) \bar{v}\|+\|\bar{v} \cdot \nabla \varphi\|+\|\operatorname{div}(\nabla \varphi \otimes \nabla \varphi)\|+\left\|\Delta W^{\prime}(\varphi)\right\| \\
\leqslant\|\bar{v}\|+\|\varphi\|+\|\bar{v}\|_{\infty} \cdot\left\|\bar{v}_{x}\right\|+\|\bar{v}\|_{\infty} \cdot\|\nabla \varphi\|+C\|\nabla \varphi\|_{\infty} \cdot\|\varphi\|_{2,2} \\
+\left\|W^{\prime \prime}(\varphi)\right\|_{\infty} \cdot\|\Delta \varphi\|+\left\|W^{\prime \prime \prime}(\varphi)\right\|_{\infty} \cdot\|\nabla \varphi\|_{\infty} \cdot\|\nabla \varphi\| .
\end{gathered}
$$

Отсюда, используя (8), (9) (теоремы вложения), а также доказанные нами оценки (18), (21), (27), сразу получаем неравенство (11). Теорема 1 доказана. 
2. Поведение решения при $t \rightarrow \infty$. Теоремы 2,3 , доказанные в этом пункте, определяют поведение решения задачи (5)-(7) при $t \rightarrow \infty$. К сожалению, не удалось установить, что решение сходится к стационарному решению задачи. Следует отметить, что этот факт пока не доказан даже для уравнения Кана-Хилларда, т.е. для уравнения (6) при $\bar{v} \equiv 0$. С исследованием этого вопроса можно ознакомиться, например, по статьям [9]-[12]. Работы [13], [14] посвящены исследованию поведения решений уравнений Навье-Стокса, хотя, предложенньй там метод применим и к другим нелинейным уравнениям.

Сначала изучим поведение функции $\bar{v}(t)$.

Теорема 2. Пусть $\bar{v}(t)$ - решение задачи (5)-(7), существование которого доказано в теореме 1 . Тогда $\|\bar{v}(t)\|_{4} \rightarrow 0,\left\|\bar{v}_{x}(t)\right\| \rightarrow 0$ при $t \rightarrow \infty$, причем

$$
\left\|\bar{v}_{x}(t)\right\| \leqslant C e^{-\gamma t}
$$

әде $C, \gamma$ - некоторые положстельные постоянные, не зависящие от $t$.

ДокАЗАТЕльСТво. В силу оценок $(24),(27),(13)$ из (26) получим

$$
\frac{d}{d t}\left\|\bar{v}_{x}\right\|^{2}+C_{1}\left\|\bar{v}_{x}\right\|^{2} \leqslant C_{2}+C_{3}\|\nabla \theta\|^{2} .
$$

Интегрируя это неравенство по $t$ от 0 до некоторого $T>0$, находим

$$
\left\|\bar{v}_{x}(T)\right\|^{2} \leqslant\left(\left\|\bar{v}_{x}(0)\right\|^{2}+\int_{0}^{T}\left(C_{2}+C_{3}\|\nabla \theta\|^{2}\right) d t\right) e^{-C_{1} T} .
$$

Отсюда следует (28). Из оценки (14) видим, что для $\|\bar{v}(t)\|_{4}$ имеет место аналогичная (28) оценка. Теорема доказана.

Теперь исследуем поведение функции $\varphi(t)$. Для этого необходимо предварительно доказать несколько утверждений.

ЛЕмма 2.1. Для любого $\delta>0$ функиия $\varphi(t)$ равномерно непрерывна по $t$ в пространстве $H^{4 \sigma}, 0<\sigma<1$, на интервале $(\delta, \infty)$, а именно,

$$
\|\varphi(t+\tau)-\varphi(t)\|_{4 \sigma, 2} \leqslant C \tau^{\lambda},
$$

әде $\lambda<1-\sigma, C$ - некоторая постоянная, зависящая только от $\sigma$ и начальных условий.

ДокАЗАТЕЛЬСтво. В силу уравнения (6) имеет место представление

$$
\varphi(t)=e^{-B\left(t-t_{0}\right)} \varphi\left(t_{0}\right)+\int_{t_{0}}^{t} e^{-B\left(t-t_{0}-s\right)} g(s) d s, \quad t>t_{0} .
$$

Поэтому

$$
\begin{aligned}
\|\varphi(t+\tau)-\varphi(t)\|_{4 \sigma, 2} \leqslant & C\left\|\left(e^{-B \tau}-I\right) e^{-B\left(t-t_{0}\right)} B^{\sigma} \varphi\left(t_{0}\right)\right\| \\
& +C\left\|B^{\sigma} \int_{t_{0}}^{t}\left(e^{-B \tau}-I\right) e^{-B\left(t-t_{0}-s\right)} g(s) d s\right\| \\
& +\left\|B^{\sigma} \int_{t}^{t+\tau} e^{-B\left(t-t_{0}+\tau-s\right)} g(s) d s\right\| .
\end{aligned}
$$


Так как $\varphi(t) \in H^{4}$ для почти всех $t>0$, то для любого $\delta>0$ существует $t_{0}<\delta$ такое, что $\left\|\varphi\left(t_{0}\right)\right\|_{4,2} \leqslant C$. Оценим в $(29)$ каждое слагаемое по отдельности:

$$
\begin{aligned}
& \left\|\left(e^{-B \tau}-I\right) e^{-B\left(t-t_{0}\right)} B^{\sigma} \varphi\left(t_{0}\right)\right\| \leqslant \frac{1}{\gamma} C \tau^{\gamma}\left\|e^{-B\left(t-t_{0}\right)} B^{\sigma+\gamma} \varphi\left(t_{0}\right)\right\| \\
& \leqslant \frac{1}{\gamma} \tau^{\gamma} C\left\|\varphi\left(t_{0}\right)\right\|_{4(\sigma+\gamma), 2}, \\
& \left\|B^{\sigma} \int_{t_{0}}^{t}\left(e^{-B \tau}-I\right) e^{-B\left(t-t_{0}-s\right)} g(s) d s\right\| \leqslant \frac{1}{\gamma} C \tau^{\gamma}\left\|\int_{t_{0}}^{t} B^{\sigma+\gamma} e^{-B\left(t-t_{0}-s\right)} g(s) d s\right\| \\
& \leqslant \frac{1}{\gamma} C \tau^{\gamma} \max _{s \in\left[t_{0}, t\right]}\|g(s)\| \int_{t_{0}}^{t}\left(t-t_{0}-s\right)^{-(\sigma+\gamma)} e^{-\left(t-t_{0}-s\right)} d s \\
& \leqslant \frac{1}{\gamma} C \max _{s \in\left[t_{0}, t\right]}\|g(s)\| \tau^{\gamma}, \quad \text { если } \sigma+\gamma<1 \text {, } \\
& \left\|B^{\sigma} \int_{t}^{t+\tau} e^{-B(t+\tau-s)} g(s) d s\right\| \leqslant C \max _{s \in[0, t]}\|g(s)\| \int_{t}^{t+\tau}(t+\tau-s)^{-\sigma} e^{-(t+\tau-s)} d s \\
& \leqslant C \max _{s \in[0, t]}\|g(s)\| \tau^{1-\sigma} .
\end{aligned}
$$

Так как $\max _{s \in[0, \infty)}\|g(s)\|<C$, то из (29), (30) следует утверждение леммы.

Из леммы 2.1 следует, что функция $\theta(t)$ непрерьвна по Гёльдеру с показателем $\lambda$ в пространстве $H^{4 \sigma-2}(0<\sigma<1)$, а значит, и в $L_{4}$, на интервале $(\delta, \infty)$.

Далее, в силу леммы $1.2\|\theta(t)\|<C$. Поэтому из $(14)$ и $(12)$ имеем $\theta \in L_{4}\left((0, \infty) \times \mathbb{R}^{2}\right)$.

ЛЕмма 2.2. $\|\theta(t)\|_{4} \rightarrow 0$ npu $t \rightarrow \infty$.

ДокАЗАТЕЛЬСТво. Используя только что сформулированные свойства функции $\theta(t)$, получаем

$$
\lim _{t \rightarrow \infty}\|\theta(t)\|_{4}^{4}=\lim _{t \rightarrow \infty} \lim _{\tau \rightarrow 0} \frac{1}{\tau} \int_{t}^{t+\tau}\|\theta(s)\|_{4}^{4} d s=\lim _{\tau \rightarrow 0} \lim _{t \rightarrow \infty} \frac{1}{\tau} \int_{t}^{t+\tau}\|\theta(s)\|_{4}^{4} d s=0 .
$$

Лемма доказана.

ОПРЕДЕЛЕНИЕ. Будем говорить, что последовательность функций $u_{k}$ локально сходится $\kappa$ функиии $u$ в пространстве $H^{p}$ и обозначать $u_{k} \stackrel{l}{\rightarrow} u$, если $u_{k} \rightarrow u$ в $H^{p}(K)$ для любого компакта $K \subset \mathbb{R}^{2}$.

Обозначим через $\omega\left(\bar{v}_{0}, \varphi_{0}\right)$ подмножество пространства $J H^{\alpha} \times H^{\beta}$, состоящее из пар функций $(\bar{v}, \varphi)$, для которых существует последовательность $t_{k} \rightarrow \infty(k \rightarrow \infty)$ такая, что $\bar{v}\left(t_{k}\right) \stackrel{l}{\rightarrow} \bar{v}$ в $L_{4}, \varphi\left(t_{k}\right) \stackrel{l}{\rightarrow} \varphi$ в $H^{\sigma}(\sigma<\beta)$, где $(\bar{v}(t), \varphi(t))-$ решение задачи $(5)-(7)$.

ТЕорема 3. Для любых $\bar{v}_{0} \in J H^{\alpha}, \varphi_{0} \in H^{\beta}, 1<\alpha<2,2<\beta<4$, мнохество $\omega\left(\bar{v}_{0}, \varphi_{0}\right)$ непусто и имеет место включение

$$
\omega\left(\bar{v}_{0}, \varphi_{0}\right) \subset\left\{(\bar{v}, \varphi) \in H^{\alpha} \times H^{\beta} \mid \bar{v}=0, \Delta \varphi-W^{\prime}(\varphi)=0\right\} .
$$


ДоказАТЕЛЬСтво. Тот факт, что $\bar{v}(t)$ стремится к 0 , доказан в теореме 1 . Исследуем поведение функции $\varphi(t)$. Так как $\|\varphi(t)\|_{\beta, 2}<C$, то множество $\{\varphi(t), t \geqslant 0\}$ компактно в $H^{\sigma}(K), \sigma<\beta$, для любого компакта $K \subset \mathbb{R}^{2}$. Поэтому множество $\omega\left(\bar{v}_{0}, \varphi_{0}\right)$ непусто. Осуществим предельный переход по любой сходящейся подпоследовательности при $t \rightarrow \infty$ в уравнении

$$
(-\Delta \varphi(t)+W(\varphi(t)), \eta)=(\theta(t), \eta)
$$

где $\eta \in C_{0}^{\infty}\left(\mathbb{R}^{2}\right)$. Из леммы 2.2 получим, что предельные точки множества $\{\varphi(t), t \geqslant 0\}$ при $t \rightarrow \infty$ должны быть решениями уравнения

$$
\Delta \varphi-W^{\prime}(\varphi)=0
$$

Теорема доказана.

ЗАмЕчАниЕ. Если бы удалось показать, что решения уравнения (31) изолированы, то решение задачи (5)-(7) сходилось бы к стационарным точкам динамической системы (5), (6).

3. Исследование устойчивости стационарных решений. Для начала докажем одно утверждение, позволяющее описать множество $S$ стационарных точек динамической системы $(5),(6)$ в $J H^{\alpha} \times H^{\beta}$.

Tеорема 4. Множество $S$ совпадает $c \cup \omega\left(\bar{v}_{0}, \varphi_{0}\right)$, әде облединение берется по всем $\left(\bar{v}_{0}, \varphi_{0}\right) \in H^{\alpha} \times H^{\beta}$.

ДокАЗАТЕЛЬСтво. Введем обозначение

$$
E(\bar{v}, \varphi)=\frac{1}{2}\|\bar{v}\|^{2}+\frac{1}{2}\|\nabla \varphi\|^{2}+\int_{\mathbb{R}^{2}} W(\varphi) d \bar{x} .
$$

В силу (12)

$$
\frac{d}{d t} E(\bar{v}(t), \varphi(t))+\|\nabla \bar{v}(t)\|^{2}+\|\nabla \theta(t)\|^{2}=0
$$

поэтому $E$ - функция Ляпунова динамической системы $(5),(6)$.

Если $\left(\bar{v}_{*}, \varphi_{*}\right) \in S$, то ясно, что $\left(\bar{v}_{*}, \varphi_{*}\right) \in \omega\left(\bar{v}_{*}, \varphi_{*}\right)$, т.е. $S \subset \cup \omega\left(\bar{v}_{0}, \varphi_{0}\right)$.

Обратно, если $\left(\bar{v}_{*}, \varphi_{*}\right) \in \omega\left(\bar{v}_{0}, \varphi_{0}\right)$ для некоторых $\bar{v}_{0} \in J H^{\alpha}, \varphi_{0} \in H^{\beta}$, то, подставив эту пару функций в уравнения $(5),(6)$, нетрудно убедиться в том, что доказываемое утверждение легко следует из равенства

$P \operatorname{div}\left(\nabla \varphi_{*} \otimes \nabla \varphi_{*}\right)=P\left(\nabla \varphi_{*} \Delta \varphi_{*}+\frac{1}{2} \nabla\left|\nabla \varphi_{*}\right|^{2}\right)=P\left(W^{\prime}\left(\varphi_{*}\right) \nabla \varphi_{*}\right)=P\left(\nabla W\left(\varphi_{*}\right)\right)=0$.

Теорема доказана.

ЗАмЕчАниЕ. Следует заметить, что множество $S$ совпадает также с множеством стационарных точек функционала Ляпунова $E$.

ЛЕмма 3.1. Для решений уравнения (31) имеет место оченка $0 \leqslant \varphi(\bar{x}) \leqslant 1$. 
ДокАЗАТЕЛЬСТво. Пусть $f: \mathbb{R} \rightarrow \mathbb{R}$ - функция, удовлетворяющая условиям

1) $f \in C^{2}(\mathbb{R})$;

2) $f^{\prime \prime}(\xi) \geqslant 0$ для любого $\xi \in \mathbb{R}$;

3) $f(\xi)=0$ при $\xi \in[0,1]$.

Домножив (31) на $f^{\prime}(\varphi)$ и проинтегрировав, получим

$$
\int_{\mathbb{R}^{2}}\left[f^{\prime \prime}(\varphi)|\nabla \varphi|^{2}+f^{\prime}(\varphi) W^{\prime}(\varphi)\right] d \bar{x}=0
$$

Отсюда, так как $f^{\prime}(\xi) W^{\prime}(\xi) \geqslant 0$ для всех $\xi \in \mathbb{R}$, то $f^{\prime}(\varphi) W^{\prime}(\varphi)=0$. Следовательно, $0 \leqslant \varphi \leqslant 1$. Лемма доказана.

ТЕОрема 5. Функиии $\bar{v} \equiv 0, \varphi \equiv 0$ образуют асимптотически устойчивое стаиионарное решение задачи (5)-(7). Все другие стационарные решения не являются асимптотически устойчивыми. Более того, если $\left(\bar{v}_{*}, \varphi_{*}\right)$-ненулевое стационарное решение задачи (5), (6) такое, что в $\mathrm{JH}^{\alpha} \times H^{\beta}$ существует окрестность точ$\kappa и\left(\bar{v}_{*}, \varphi_{*}\right)$, в которой нет других стационарных точек $(\bar{v}, \varphi)$, удовлетворяющих условию $E(\bar{v}, \varphi)<E\left(\bar{v}_{*}, \varphi_{*}\right)$, тогда $\left(\bar{v}_{*}, \varphi_{*}\right)$ - неустойчивое решение.

ДоказАтЕЛЬСтво. Тот факт, что $(0,0) \in S$, проверяется непосредственно. Приступим к исследованию устойчивости. Так как $d E(\bar{v}, \varphi) / d t<0$ вне стационарных точек, то для исследования устойчивости достаточно исследовать тип стационарных точек функционала $E$.

Линеаризуем уравнение (31) на точном решении $\varphi$ :

$$
\Delta u-W^{\prime \prime}(\varphi) u=0
$$

Заметим, что $W^{\prime \prime}(\varphi)=12 \varphi^{2}-12 \varphi+2$. Из леммы 3.1 следует, что $-1 \leqslant W^{\prime \prime}(\varphi) \leqslant 2$, причем так как $W^{\prime \prime}(0)=2$, то $W^{\prime \prime}(\varphi(\bar{x})) \rightarrow 2$ при $|\bar{x}| \rightarrow \infty$.

ЛЕмма 3.2. $\sigma_{\mathrm{ess}}\left(-\Delta+W^{\prime \prime}(\varphi(\bar{x}))\right)=[2, \infty)$, где $\sigma_{\mathrm{ess}}(\mathbb{R})-$ существенный спектр onepamopa $R$.

ДокАЗАТЕЛЬСтво. Введем обозначение

$$
m(\bar{x})=W^{\prime \prime}(\varphi(\bar{x}))-2=12 \varphi^{2}(\bar{x})-12 \varphi(\bar{x})
$$

Так как $\varphi \in L_{2} \cap L_{\infty}$, то $m \in L_{2} \cap L_{\infty}$. Но $\sigma(-\Delta+2)=\sigma_{\text {ess }}(-\Delta+2)=[2, \infty)$, где $\sigma(R)$ - спектр оператора $R$. В силу того, что оператор умножения на функцию $m$ относительно компактен по отношению к оператору $(-\Delta+2)$, то по теореме Вейля $[15$, гл. XIII]

$$
\sigma_{\mathrm{ess}}(-\Delta+2+m(\bar{x}))=\sigma_{\mathrm{ess}}(-\Delta+2) .
$$

Лемма доказана.

ЛЕмма 3.3. Нулевое решение задачи (5)-(7) асимптотически устойчиво. 
ДокАЗАТЕЛЬСТво. Так как $W^{\prime \prime}(0)=2$, топри $\varphi(\bar{x}) \equiv 0-\Delta+W^{\prime \prime}(\varphi(\bar{x}))=-\Delta+2$. Но $\sigma(-\Delta+2)=[2, \infty)$, т.е. спектр строго положителен. Поэтому $\varphi(\bar{x}) \equiv 0$-устойчивое решение. Лемма доказана.

Нами доказана первая часть теоремы. Теперь исследуем ненулевые стационарные решения.

ЛЕмма 3.4. Если решение уравнения (31) $\varphi(\bar{x}) \not \equiv 0$, mо $\sigma_{\mathrm{disc}}\left(-\Delta+W^{\prime \prime}(\varphi(\bar{x}))\right)$ непуст. В частности, 0 является собственным числом, кратность которого не меньие 2 .

ДокАЗАТЕЛЬСтво. Очевидно, что функции $\partial \varphi / \partial x_{1}, \partial \varphi / \partial x_{2}$ являются решениями уравнения (32), откуда и следует утверждение леммы.

Очевидно, что если $(\bar{v}(\bar{x}), \varphi(\bar{x}))$ - ненулевое стационарное решение задачи $(5),(6)$, то имеется целое многообразие стационарных решений $\left\{(\bar{v}(\bar{x}), \varphi(\bar{x}+\bar{a})), \bar{a} \in \mathbb{R}^{2}\right\}$. То есть в любой окрестности ненулевой стационарной точки существуют другие стационарные точки. Следовательно, любое ненулевое стационарное решение не является асимптотически устойчивым.

Дальнейший план доказательства состоит в следующем. Согласно теореме XIII.44 из [15], если $H$ - самосопряженный ограниченньй снизу оператор, $\sigma_{\operatorname{disc}}(H)$ непуст и оператор $e^{-t H}$ усиливает положительность для всех $t>0$, то $\lambda_{1}=\inf \sigma(H)$ - простое собственное значение.

Мы докажем, что оператор $e^{-t H}$, где $H=-\Delta+W^{\prime \prime}(\varphi(\bar{x}))+1$, усиливает положительность. Из леммы 3.4 следует, что 1 - собственное число оператора $H$ кратности 2 , поэтому 1 не может быть его наименьшим собственным числом. То есть у оператора $\left(-\Delta+W^{\prime \prime}(\varphi(\bar{x}))\right)$ существует отрицательное собственное число. Отсюда будет следовать последнее утверждение теоремы.

ЛЕмма 3.5. Пусть задань функиии $m_{i} \in L_{\infty}, i=1,2, u_{0} \in L_{2}$ такие, что $0 \leqslant m_{1} \leqslant m_{2}, u_{0} \geqslant 0$. Тогда $u_{1} \geqslant u_{2}$, где $u_{i} \in H^{2}, i=1,2$, есть решение задачи

$$
u_{i t}=\Delta u_{i}-m_{i} u_{i}, \quad u_{i}(0)=u_{0}
$$

ДокАЗАТЕЛЬСТВо. Обозначим $u=u_{1}-u_{2}, m=m_{1}-m_{2}$. Тогда

$$
u_{t}=\Delta u-m_{2} u-m u_{1}, \quad u(0)=u_{0}
$$

В силу принципа максимума $u_{i} \geqslant 0, i=1,2$, поэтому $m u_{1} \leqslant 0$, а следовательно, $u \geqslant 0$. Лемма доказана.

Лемма 3.6. Oператор $e^{-t H}$, где $H=-\Delta+W^{\prime \prime}(\varphi(\bar{x}))+1$, усиливает положительность для всех $t>0$, т.е. если $u_{0} \geqslant 0$, то $e^{-t H} u_{0}>0$.

ДокАЗАТЕЛЬСтво. Пусть $u_{0} \geqslant 0$ - некоторая функция из $L_{2}$. Тогда $u(t)=e^{-t H} u_{0}$ есть решение задачи

$$
u_{t}=\Delta u-W^{\prime \prime}(\varphi(\bar{x})) u-u, \quad u(0)=u_{0}
$$


Рассмотрим функцию $w(t)$, являющуюся решением задачи

$$
w_{t}=\Delta w-3 w, \quad w(0)=u_{0}
$$

Из леммы 3.5 следует, что $u \geqslant w$, так как $0 \leqslant W^{\prime \prime}(\varphi(\bar{x}))+1 \leqslant 3$. Но функция $w(t)$ строго положительна при всех $t>0$. Это следует из явного представления решения задачи (33):

$$
w(\bar{x}, t)=\frac{e^{-3 t}}{4 \pi t} \int_{\mathbb{R}^{2}} u_{0}(\bar{\xi}) e^{-|\bar{x}-\bar{\xi}|^{2} /(4 t)} d \bar{\xi}>0
$$

Лемма доказана.

Итак, если $\left(\bar{v}_{*}, \varphi_{*}\right) \in S$ и $\varphi_{*} \not \equiv 0$, то оператор $\left(-\Delta+W^{\prime \prime}\left(\varphi_{*}\right)\right)$ имеет отрицательное собственное число. В силу полученной гладкости функции $\varphi$ нетрудно убедиться в том, что соответствующий собственный вектор $\psi$ принадлежит, по крайней мере, пространству $H^{4}$. Тогда для любого $\varepsilon>0$ найдется функция $\psi_{\varepsilon},\left\|\psi_{\varepsilon}\right\|_{4,2} \leqslant \varepsilon$, для которой

$$
E\left(\bar{v}_{*}, \varphi_{*}+\psi_{\varepsilon}\right)<E\left(\bar{v}_{*}, \varphi_{*}\right) .
$$

Заметим, что так как $\left(\bar{v}_{*}, \varphi_{*}\right) \in S$, то $\bar{v}_{*} \equiv 0$.

Обозначим через $U_{t}\left(\bar{v}_{0}, \varphi_{0}\right)$ полугруппу с параметром $t$, соответствующую задаче (5)-(7). Так как

$$
\frac{d}{d t} E\left(U_{t}(\bar{v}, \varphi)\right) \leqslant 0
$$

для любых $\bar{v} \in J H^{\alpha}, \varphi \in H^{\beta}$, то

$$
E\left(U_{t}\left(\bar{v}_{*}, \varphi_{*}+\psi_{\varepsilon}\right)\right) \leqslant E\left(\bar{v}_{*}, \varphi_{*}+\psi_{\varepsilon}\right), \quad t \geqslant 0 .
$$

Следовательно, используя (34) и лемму Фату, для любых $(\bar{v}, \varphi) \in \omega\left(\bar{v}_{*}, \varphi_{*}+\psi_{\varepsilon}\right)$ получаем

$$
E(\bar{v}, \varphi)<E\left(\bar{v}_{*}, \varphi_{*}\right)
$$

Пусть $S_{1}=\left\{(\bar{v}, \varphi) \in S \mid E(\bar{v}, \varphi) \geqslant E\left(\bar{v}_{*}, \varphi_{*}\right)\right\}$. Обозначим через $B_{r}$ замкнутьй шар в $H^{\alpha} \times H^{\beta}$ радиуса $r$ и с центром в точке $\left(\bar{v}_{*}, \varphi_{*}\right)$ такой, что $B_{r} \cap\left(S \backslash S_{1}\right)=\varnothing$. Такой шар существует по условию теоремы.

В силу (35) и теоремы 4 имеем $\omega\left(\bar{v}_{*}, \varphi_{*}+\psi_{\varepsilon}\right) \cap B_{r}=\varnothing$.

Отсюда следует, что для любого $n>0$ существует $t>n$ такое, что $U_{t}\left(\bar{v}_{*}, \varphi_{*}+\psi_{\varepsilon}\right) \notin B_{r}$. Это дает нам неустойчивость стационарного решения $\left(\bar{v}_{*}, \varphi_{*}\right)$, так как $\varepsilon$ произвольно и $r$ от $\varepsilon$ не зависит. Теорема доказана.

Автор выражает благодарность П.И. Плотникову за полезные обсуждения работы. 


\section{СПИСОК ЦИТИРОВАННОЙ ЛИТЕРАТУРЫ}

[1] Старовойтов В. Н. Модель движения двухкомпонентной жидкости с учетом капиллярных сил // Журн. прикл. мех. и техн. физики. 1994. №6. С. 61-71.

[2] Van der Waals J. D. Thermodynamics theory of capillarity flow under the hypothesis of a continous variation of density // Verhandel. Konink. Akad. Weten. Amsterdam. Sec. 1. 1893. V. 1. № 8 .

[3] Korteweg D. J. Sur la forme que prennent les équations du mouvement des fluides si l'on tient compte des forces cappillaires causées par des variations de densité // Archives Neerlandaises des Sciences Exactes et Naturelles. Ser. II. 1901. V. 6. P. 1-24.

[4] Cahn J. W., Hilliard J. E. Free energy of a non-uniform system. Interfacial free energy // J. Chem. Phys. 1958. V. 28. P. 258-267.

[5] Трибель Х. Теория интерполяции, функциональные пространства, дифференциальные операторы. М.: Мир, 1980.

[6] Соболевский П.Е. О нестационарньх уравнениях гидродинамики вязкой жидкости // Докл. АН СССР. 1959. Т. 128. №1. С. 45-48.

[7] Хенри Д. Геометрическая теория полулинейных параболических уравнений. М.: МИР, 1985.

[8] Ладыженская О.А. Математические вопросы динамики вязкой несжимаемой жидкости. М.: Наука, 1970.

[9] Калантаров В.К. О глобальном поведении решений некоторых нелинейных уравнений четвертого порядка // Записки научных семинаров ЛОМИ. Краевые задачи математической физики и смежные вопросы теории функций. 1987. Т. 163. С. 66-75.

[10] Elliot C. M., Songmu Z. On the Cahn-Hilliard equation // Arch. Rational Mech. Anal. 1986. V. 96. P. 339-357.

[11] Nicolaenko B., Scheurer B. Low dimensional behaviour of the pattern formation Cahn-Hilliard equation // Trends and practice of Nonlinear Analysis. North Holland: Elsevier Sci. Publ., 1985. P. 323-336.

[12] Alikakos N.D., Fusco G. Slow dynamics for the Cahn-Hilliard equation in higher space dimensions. Spectral estimates // Comm. Partial Differential Equations. 1994. V. 19. P. 1397-1447.

[13] Ладыженская О.А. О динамической системе, порождаемой уравнениями Навье-Стокса // Записки научных семинаров ЛОМИ. 1972. Т. 27. С. 91-114.

[14] Ладыженская О. А. О нахождении минимальных глобальных аттракторов для уравнений Навье-Стокса и других уравнений с частными производными // УМН. 1987. Т. 42. № 6(258). C. 25-60.

[15] Рид М., Саймон Б. Методы современной математической физики. Т. 4. Анализ операторов. М.: Мир, 1982.

Институт гидродинамики им. М. А. Лаврентьева СО РАН, 